Revue

d'ethnoécologie
Revue d'ethnoécologie

$7 \mid 2015$

Varia + dossier "Anthropologie de la conservation"

\title{
Des insectes et des hommes (Nord du Cameroun)
}

of insects and men (North Cameroon)

\section{Christian Seignobos}

\section{(2) OpenEdition}

Journals

Édition électronique

URL : http://journals.openedition.org/ethnoecologie/2180

DOI : 10.4000/ethnoecologie.2180

ISSN : 2267-2419

Éditeur

Laboratoire Eco-anthropologie et Ethnobiologie

Référence électronique

Christian Seignobos, «Des insectes et des hommes (Nord du Cameroun) 》, Revue d'ethnoécologie [En ligne], 7 | 2015, mis en ligne le 30 juin 2015, consulté le 19 avril 2019. URL : http:// journals.openedition.org/ethnoecologie/2180 ; DOI : 10.4000/ethnoecologie.2180

Ce document a été généré automatiquement le 19 avril 2019

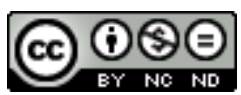

Revue d'ethnoécologie est mis à disposition selon les termes de la licence Creative Commons Attribution - Pas d'Utilisation Commerciale - Pas de Modification 4.0 International. 


\section{Des insectes et des hommes (Nord du Cameroun)}

of insects and men (North Cameroon)

Christian Seignobos

1 La focalisation des Mofu des monts Mandara sur leurs insectes, que nous avions mise en évidence (Seignobos et al. 1996) pouvait se satisfaire d'une explication, celle de leur situation d'« Hommes des rochers", comme ils se désignaient eux-mêmes, privés de brousse et d'accès à la plaine. Ils n'avaient d'animaux que ceux de leur élevage et pour seule faune sauvage, les insectes. Cette singularité mofu demandait toutefois à être étayée par une démarche comparatiste auprès d'autres groupes touchant à leurs rapports avec l'entomofaune. C'est une partie des résultats de cette enquête (courant 2011) que nous présentons.

2 Les insectes constituent un vaste bestiaire - mais il n'est pas l'unique - dans lequel les populations puisent pour leurs contes, leurs proverbes, leurs injures et pour maints raccourcis du langage illustrant rapports de force, traits de caractère et situations particulières. La pensée analogique fonctionne à plein pour faire des insectes le miroir de la société humaine ${ }^{1}$.

3 La mise en parallèle avec la nomenclature zoologique scientifique présente des cas surprenants de similitudes, mais bien d'autres en revanche renvoient à des structures cognitives étrangères à sa logique ${ }^{2}$. Toutefois chez les Mofu nord, par exemple, 70 \% des arthropodes nommés présentent une association forte entre nom mofu et taxon scientifique (Aberlenc \& Deguine 1999 : 115). S'il existe une volonté de produire une grille classificatoire, elle n'est qu'ébauchée et reste très ouverte. Un seul ordre, celui des orthoptères, semble avoir ses composants nommés pour la plupart. Le reste renvoie à des formes de regroupements souvent incomplets. Pour rester chez les Mofu nord : dladlak s'applique à tous les termites ailés et aux fourmis ailées du genre Dorylus; va'al regroupe des organismes hématophages de même taille, punaises de lit et tiques du chien; tol désigne les chenilles et les vers non comestibles souvent déprédateurs des récoltes 
(Aberlenc \& Deguine 1999). Ailleurs, ce ne seront pas les mêmes regroupements, ceux porteurs de carapaces et qui volent sont les neverekna chez les Masa; les Tupuri intègrent dans la catégorie gwoko tout ce qui vole, au sens large, coccinelles, abeilles (Apidae), trigones, alors que celle de gungun (comme vengavenga chez les Masa) regroupe cétoines, buprestes et longicornes. Les Kapsiki désignent par vulumu tous les insectes vivant sur terre ou dans l'eau, mais susceptibles d'envol. On peut observer des groupements particuliers, par exemple pour les insectes foreurs, fotna en masa, qui mêle bruches, charançons, mais aussi un xylocope, une grosse abeille charpentière. Les insectes sociaux dans le sol, les termites et fourmis, et dans le ciel, abeilles, trigones... n'ont pas de termes génériques, et renvoient à des groupes d'insectes présentés comme des « familles » par nos informateurs.

Ces classifications peuvent également changer au sein du même groupe ethnique et aussi d'une génération à l'autre. Avec le développement de la scolarisation et les divers enseignements délivrés auprès des groupements paysans par l'encadrement des sociétés paraétatiques, le cycle biologique chenille-chrysalide-papillon a été adopté, encore que certaines chenilles portant un nom ne soient pas associées à leur imago. Les vieux adultes désignent certaines chenilles comme formant une «famille». Dans le passé, le stade larvaire des insectes intéressait autrement plus les hommes comme aliment ou en tant que ravageurs que les papillons. On attribue à ces derniers des noms génériques traduisant le plus souvent une onomatopée ${ }^{3}$. Ces insectes dérisoires sont qualifiés de «poulets de Dieu » (gogor ma bwi erlam) ou de "poulets de la panthère » (gogor ma duvar) évoquant ainsi les ocelles et ce chez les groupes Rhide comme chez les Mofu. À l'exception des Mofu du massif de Mbokou du quartier de Giriya-Maya où un groupe parti de Mowo centre suivit un énorme papillon (Saturniidae) jusqu'au massif de Mbokou où il entra dans la terre. Lorsqu'il en sortit ses battements d'ailes firent s'enfuir les nuages porteurs de pluies. Les Guriya, maîtres de la pluie, associent encore aujourd'hui à leur combinaison rituelle visant à convoquer la pluie ce groupe qui sacrifie à « leur » papillon. L'onomatopée peut aussi servir à désigner des insectes gustativement très prisés comme les Ruspolia spp. : mekwete-kwete chez les Mofu Gudur (Barreteau 1999 : 138) et motwot(k)woteng pour les Mofu nord, qui lui ont également conservé son nom de guslen. Un grand nombre d'insectes se trouve indexé à un végétal, arbre ou phorbe sur lequel ils sont censés se trouver, mais aussi à des cultures, sorghos, oseille de Guinée... voire des paléocultures. Chez les Mofu, Stenocoris (tek marta, «la chose de l'éleusine» Eleusine coracana), une punaise alydide, et d'autres insectes sur l'indigotier sont aujourd'hui passés sur de nouvelles cultures hôtes.

5 La dénomination des insectes concerne forcément ceux jugés "serviables" pour l'Homme, comme nourriture, pour des soins ou par leur rôle dans la chiromancie et certains rituels, voire pour des utilisations oubliées, ou à l'inverse, dans quelques cas, pour signaler leur nocivité.

6 Nous comparerons les rapports entre insectes et sociétés à travers les discours de quelques communautés des monts Mandara et d'autres de la plaine du Logone. Les sociétés musulmanes, qui portent sur les insectes un regard moins intéressé et plus généraliste, ne sont pas concernées ${ }^{4}$. 


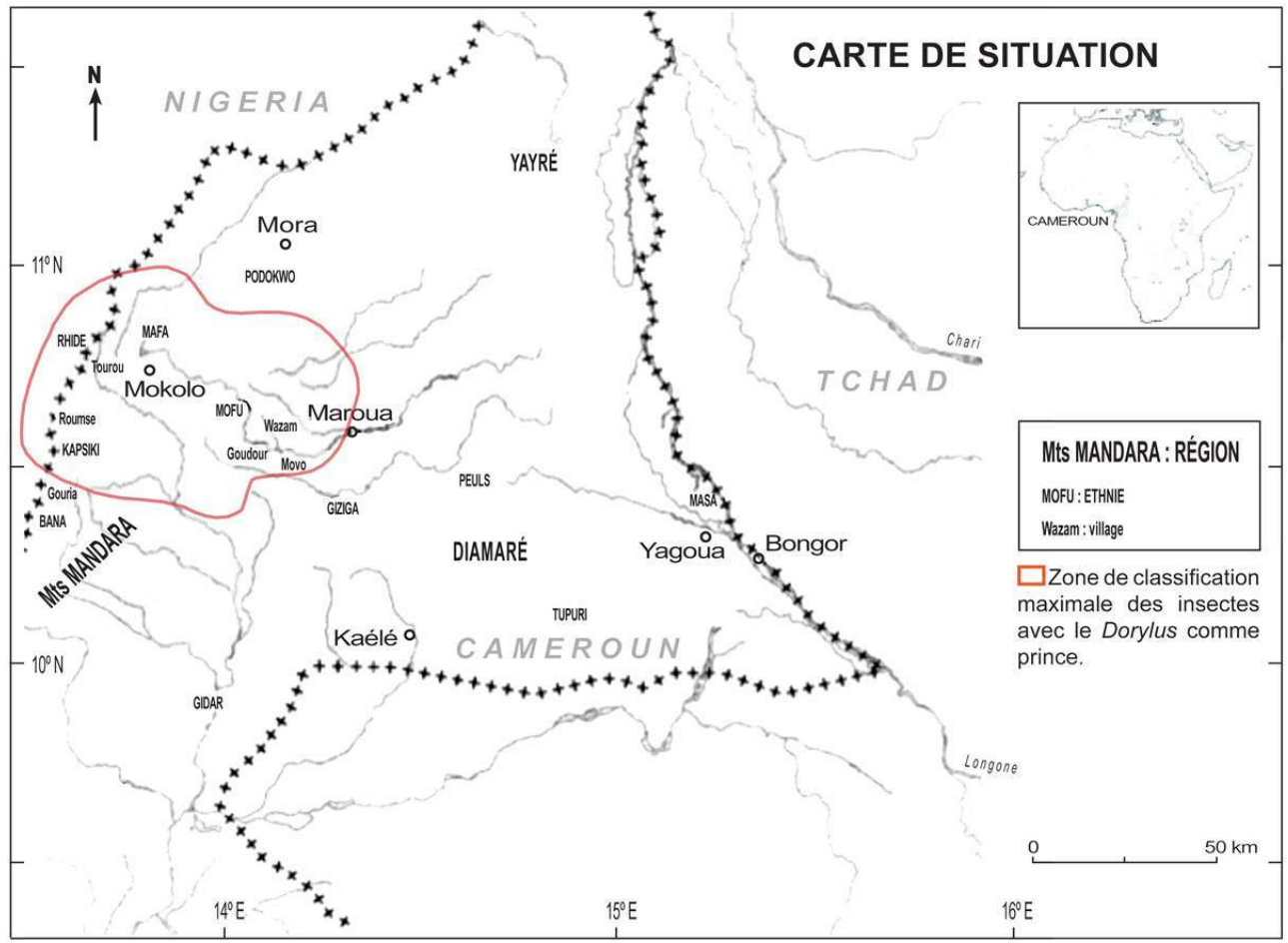

\section{Les Mandara, montagnes et plateaux}

7 Les plus « montagnardes » des ethnies occupent les reliefs enclavés (Mafa) et les massifs de bordure, jadis étroitement assiégés (Mofu). Mafa et Mofu ont été désignés par l'administration coloniale comme les "pauvres du Mandara ». Avec leurs voisins Rhide, Wula, Kapsiki, ils se révèlent des céréaliculteurs passionnés. Aussi partagent-ils le même arsenal de rituels visant à écarter sécheresse et déprédateurs afin de préserver la croissance des mils et des sorghos jusqu'à la récolte. Cet état de nécessité dans lequel ils se trouvaient aurait-il favorisé leur intimité avec les insectes?

\section{Les Mofu nord}

De Wazan à Meri, ils manifestent l'expression la plus accomplie des rapports hommesinsectes dans ce qu'ils ont de plus anthropocentrés. Il ne s'agit pas de la procédure classique d'une anthropologie cognitive concernant les insectes passant par leur identification, leur catégorisation et impliquant des niveaux de savoirs. Cette «nomenclature » relève d'un autre registre, celui d'une projection de la société des hommes sur celle des insectes à travers leurs hiérarchies et leurs pratiques sociales. Elle n'intéresse qu'une partie des insectes, ceux signifiants seront alors particulièrement observés voire, pour certains, scrutés.

Parmi les fourmis, jaglavak, une fourmi légionnaire de la sous-famille des Dorylinae est qualifiée de Prince des insectes. Il est utilisé pour chasser les termites et autres insectes indésirables qui envahissent les toitures et les silos des habitations. Aucun insecte ne saurait lui résister, aussi les Mofu lui vouent-ils une admiration sans bornes (Seignobos 1999). Son adjoint, singel gagazana, est une fourmi de la sous-famille des Myrmicinae. Les chefs de quartiers sont mazaza (Camponotus maculatus), mangirmak (Pachycondyla sp.), 
singel noir... Jaglavak a aussi, sur les piémonts, un délégué mananeh (les termites Microcerotermes solidus ou Ancistrotermes crucifer).

Les serviteurs du chef sont la fourmi gula (Megaponera) ou encore ndroa (une Lepisiota). Les "pauvres", mbidlew, renvoient à la multitude des autres insectes, libellules, bousiers, myriapodes inclus.

11 La vie des insectes est calquée sur celle de la société mofu. On y retrouve les rapports entre parents paternels et maternels, les alliances matrimoniales... qui engendrent des comportements complexes d'évitement et de respect. Les relations d'amitié institutionnalisée apparaissent également. « L'intime », celui appelé ndaw kuli (« Homme du sacrifice », qui remplace le chef de famille empêché pour célébrer à sa place les rituels chez lui) est, pour jaglavak, mananeh, alors que le ndaw kuli de mananeh n'est autre que ndroa... On comprend que l'imbrication des relations tissées entre les insectes et les comportements qui leur sont prêtés au plus près de ceux des Mofu soient pour eux un constant sujet d'observation. Le termite mananeh vit plutôt en plaine et jaglavak l'évite comme il évite un autre insecte social, la fourmi gula. En cas de conflit, l'un ne saurait l'emporter sur l'autre (Seignobos et al. 1996 : 131, 132).

Figure 1

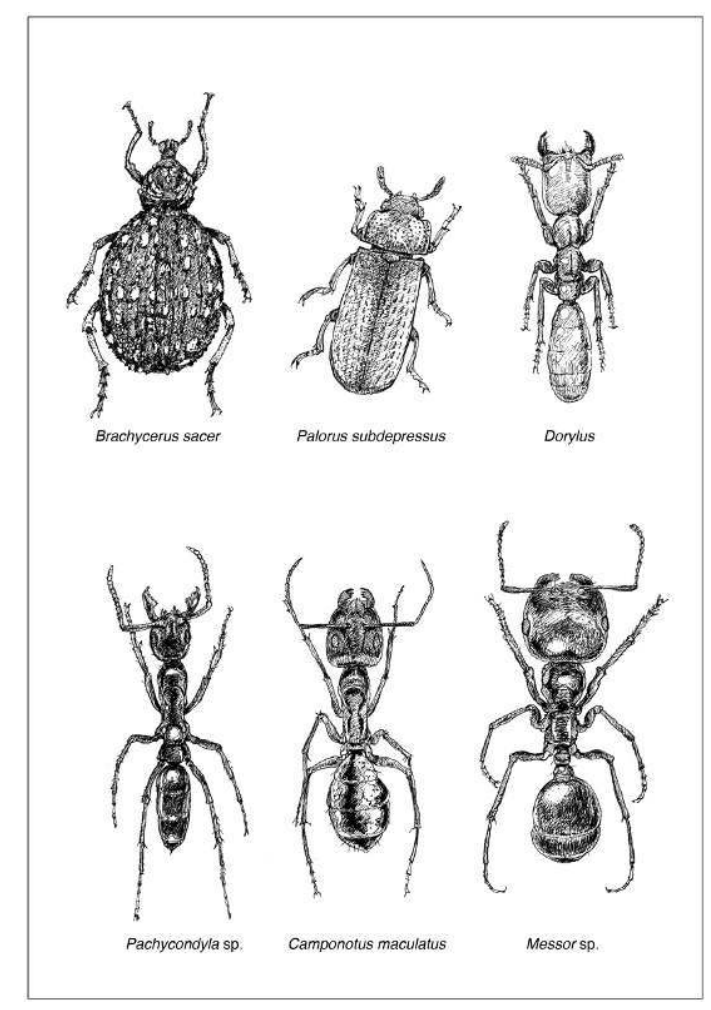

Dessin C. Seignobos

Lorsque les insectes manifestent des comportements « incestueux » ou déviants et que, par exemple, jaglavak attaque mananeh, ce pouvait être, encore au début $d u x^{e}$ siècle, un signe majeur qui sera rapporté à la chefferie et dont on cherchait la signification avec l'appui de tout ce que le massif comptait de devins. 


\section{Les Mafa et les Rhide}

Le grand groupe voisin des Mofu présente des comportements assez semblables envers les insectes. Gwendjereng, la fourmi processionnaire/légionnaire (Dorylinae) en est toujours le chef, avec ses notables comme la fourmi Messor et aussi les multiples apparentements. À la différence des Mofu Nord, les chefs mafa sont peu puissants. En revanche, un élément clef de la société est le forgeron casté (gwalda), absent chez les Mofu Nord. Le monde des insectes comptera donc un chef forgeron, en l'occurrence la fourmi marbaba, personnage qui se retrouvera également dans les monts Mandara au sud du pays mafa.

De région de Tourou, chez les Rhide, la fourmi doryline rhidsing demeure le chef des insectes, mais ici on est davantage en présence d'une classification " sociale » avec trois personnages: le chef, le second et le forgeron, ainsi que des éléments annexes aux positionnements moins stricts.

Chez les criquets, par exemple, le plus gros Homoxyrrhepes punctipennis (vogwam) est le chef, le second est Anacridium melanorhodon (vidim) et le forgeron est Pyrgomorpha vignaudii (bidi ndrah). Dans la "grande famille» des minjil qui recouvre de nombreuses cétoines caractéristiques de cette partie des monts Mandara, Pseudoprotaetia stotala ( vurndilrhe), une cétoine mouchetée noir sur blanc et qui passe la nuit sur des matières en décomposition, en est le forgeron.

Figure 2

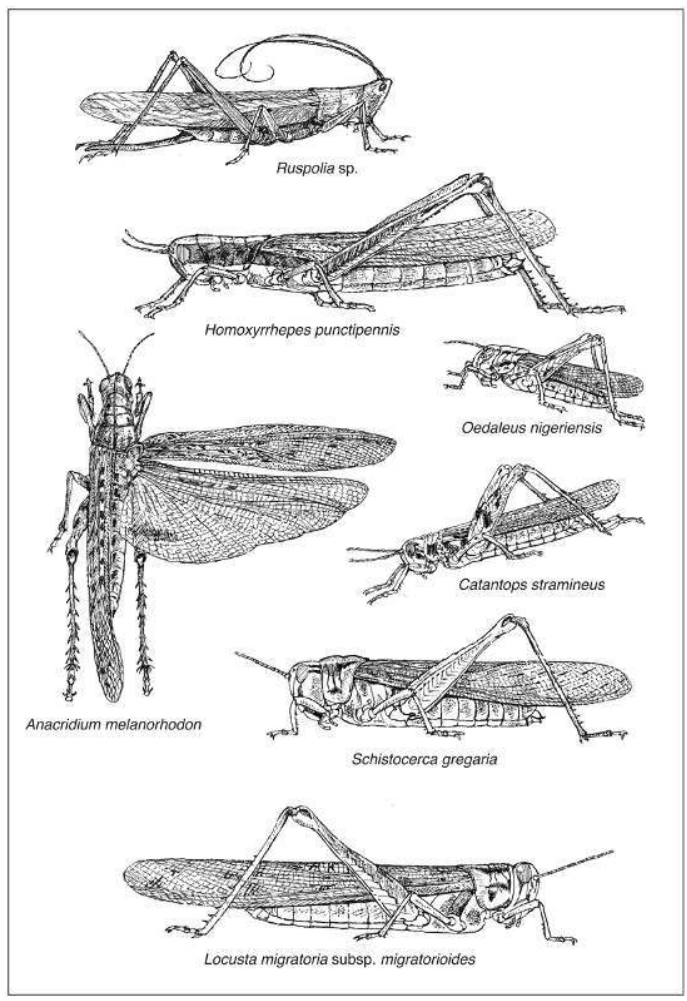

Dessin C. Seignobos 


\section{Les Kapsiki}

16 Dans la société kapsiki, chaque clan ou grand lignage (folrha) dispose de ses propres forgerons. Cette société est fondamentalement divisée entre les gens libres et les forgerons, qui ont été disqualifiés de l'accès au pouvoir parce qu'ils sont impurs, leur impureté étant directement indexée à leur obligation d'enterrer les autres. Cette mise à l'écart sociale se traduit par une endogamie stricte. Elle les contraint à des comportements d'évitement : on ne boit, ni ne mange avec les forgerons. Écartés, dans le passé, de l'accès à la terre, ils devaient échanger le fer et les poteries confectionnées par leurs femmes contre du mil. Le discours ambiant les traite d'insatiables, de gloutons consommant tout ce que les autres refusent - de parasites... défauts dont sont affublés les insectes les représentant, ce qui, par ailleurs, va à l'encontre des discours laudatifs envers ces mêmes forgerons, fondements des institutions.

Mano la fourmi doryline reste le chef des insectes ou seulement celui des non volants. À Roumze on trouve, comme second, le charançon Brachycerus sacer (shilan), réputé pour sa méchanceté - dont nous aurons à reparler. Ailleurs, c'est la fourmi Messor (kagj'a), la petite fourmi Lepisiota la secondant. Parmi les grands notables, le criquet pèlerin ( Schistocerca gregaria), dzale, aujourd'hui disparu, a été remplacé dans son rôle par une guêpe maçonne, nbriji. Le chef forgeron est ici Anacridium melanorhodon, yolama kwa rhe , cet insecte se nourrissant de tout sans interdit alimentaire, tout comme le forgeron.

Chez les criquets (rhegi), le forgeron (Catantops axillaris), kwa mbra ne peut être mangé, ni même attrapé par les enfants. Pachnoda marginata ssp. aurantia (ba jora) qui, par sa couleur brun rouge mat rappelle les peaux de chèvre cousues sur le cadavre, sera ici le forgeron des Cetoniinae. Le taupin Tetralobus flabellicornis (dlerhe) sera, à cause de l'odeur qu'il dégage, celui des Dytiscidae. Une Pheidole (mcancrhe), fourmi de la sous-famille des Myrmicinae, bien connue pour son attirance immodérée pour le sucre, sera le forgeron de la famille des fourmis dans laquelle le chef est la grosse fourmi Messor (mazentili).

Les Kapsiki aiment à illustrer leurs institutions par des images prises dans le monde des insectes. L'une d'elle est récurrente, celle de l'envol des imagos de termites au début de la saison des pluies auquel ils identifient leurs propres initiés (gola), à la même période, qui vont entrer dans la vie adulte.

On retrouve aussi chez les Kapsiki des renvois à la numérotation des enfants, de un à onze, pour les garçons comme pour les filles. Ils associent à cet ordre d'arrivée des traits de caractères, l'un est têtu, l'autre soumis ou sérieux... et ces comportements sont appliqués à certains insectes. Le huitième (kwada) compte peu, aussi donnera-t-on ce nom à un insecte sans importance comme le termite kwada cemba. La quatrième fille ( kwegne) est réputée désordonnée, aussi kwegne dra (Oedaleus senegalensis) sera un petit criquet au sautillement confus et qui n'est pas consommé. La classification oncle/tante, paternel/maternel se retrouve également chez les Kapsiki, le plus souvent au sein des mêmes « familles ».

\section{Les Giziga Bwi Marva des piémonts des Mandara}

21 Chez les Giziga voisins des montagnards mofu il semblerait que la «classification » des insectes se soit pour l'essentiel reportée sur les criquets 5 . Elle n'en reflète pas moins leur société jadis fortement structurée autour d'une puissante chefferie, Marva, qui devait 
être subjuguée par la conquête peule au début du XIX ${ }^{e}$ siècle. Mais ce transfert métaphorique embrasse plus largement l'histoire du royaume Bwi Marva et les rapports avec ses voisins. Bien que vaincus et dispersés, les Giziga Bwi Marva pouvaient entretenir la mémoire de leur histoire par le biais de cet ordre d'insectes. Les associations de criquets représentent les principaux clans giziga qui chacun disposent de leur grand notable et de leurs esclaves, ainsi que des chefferies voisines qui, toutes, obéissent à une forme de hiérarchie. Celle-ci repose en grande partie sur des comportements réels ou prétendus des criquets eux-mêmes, sur leurs tailles et leurs apparences morphologiques.

Le criquet arboricole mavay (Anacridium melanorhodon) serait toujours suivi du criquet sombre jojobiyok (Zacompsa festa?), désigné comme son esclave. Il se tient en effet toujours près de mavay, mais en retrait, jamais sur la même branche et on le capture aisément. Un autre criquet arboricole, mbuzlongor (Acanthacris ruficornis citrina) est aussi un chef et ses serviteurs sont les petits criquets mef'yew (Heteracris sp., Cataloipus sp., Harpezocatantops stylifer...).

23 Katsakara a bwi zumaya ( « le coutelas du chef zumaya »), Acrotylus sp. ?, fait référence au royaume zumaya, concurrent de Marva qui s'étendait plus en plaine. Ce criquet terricole se rencontre dans les riz sauvages. Ses esclaves sont les criquets agumer. Mu'bzlar wandala (Hieroglyphus daganensis) vole et se pose pour immédiatement repartir, et la personne qui le poursuit s'expose à s'égarer. Cette figure de mythe était largement répandue chez les Giziga où, lors de famines, les gens qui s'éloignaient trop en plaine à la poursuite de criquets étaient capturés par des cavaliers du Wandala, dominant la région avant la conquête peule. Les dépendants de mu'bzlar wandala sont l'ensemble des petits criquets dits mazlbadak.

Majuway (Ornithacris turbida) passe pour être leur chef supérieur, qui a pu représenter le chef bwi marva lui-même avec son cortège de criquets notables et serviteurs. La liste des groupes de criquets n'est pas exhaustive. Ces criquets-chefs appartiennent pour la plupart à des espèces susceptibles de se mobiliser en essaims nuisibles et de se révéler potentiellement redoutables pour les cultures, tout comme les puissants chez les Giziga avaient une réputation de voracité.

Le criquet matawtawa (Cyrtacanthacris aeruginosa?) peut venir épisodiquement de la plaine par essaims pendant la saison sèche. Ils occupent seuls les mêmes espaces, laissant après leur passage une terre enrichie de leurs déjections. Les autres criquets n'osant se mêler à eux, ils deviennent un groupe d'étrangers socialement supérieurs.

Le criquet migrateur zaray est le chef suprême de tous les criquets (bwi mada'dawe hoyokhay) ${ }^{6}$. Il s'agit de Locusta migratoria migratorioides et de ses notables Schistocerca gregaria et Oedaleus senegalensis. Ils viennent du nord, comme du royaume du Wandala dont la chefferie Bwi Marva était la vassale.

Certains criquets représentent d'autres institutions giziga, comme le chef de terre, le masahay (hoyok muwa, "criquet/montagne»). Catantops stramineus est un criquet xérophile qui s'éloigne rarement des collines rocheuses auprès desquelles se sont réfugiés les Giziga. Un autre petit criquet également xérophile, modogozlon (Oedaleus nigeriensis), au vol mesuré mais particulièrement agité et bruyant, est désigné comme le Bwi Gawla, le chef de guerre des petits criquets (hoyok basay, (Oxycatantops?) hoyok magamak et tous les mef'yew). Devant l'arrivée inopinée d'un intrus, il donne l'alarme et met en branle toute la gent des sauterelles et criquets ${ }^{7}$. 
Avec le temps et selon les contes ou les fabliaux, les criquets ont pu acquérir une seconde identité, voire plusieurs. Majuway, le criquet "rouge» (plutôt marron) représente aujourd'hui le plus souvent les Peuls. Il devint le lamido (chef peul) des criquets après l'arrêt des invasions d'essaims de zaray venus du haut Sahel à la fin des années 1930, alors que le criquet m'buzlongor devenait le chef giziga. Mavay reste encore celui des Mofu et plus précisément le chef du groupe Mowo. Ces classifications qui, par le passé, furent un miroir du monde politique, social et religieux des Giziga, évoluent peu à peu vers un simple jeu pour les jeunes bergers comme on peut en rencontrer parmi les communautés des plaines du Logone.

\section{Les plaines du Logone}

Les grands groupes, Masa et Tupuri, partagent des milieux sans comparaison avec ceux évoqués pour les ethnies des monts Mandara. Leur espace n'est pas contraint et leurs ressources infiniment plus variées. Leurs histoires comme leurs vécus, passés ou actuels, ne peuvent encore que les différencier.

\section{Les Masa}

Leur société s'est passée de pouvoirs dynastiques, seuls sont reconnus des chefs d'enclos ( bum zina), riches de leur seule famille et de leurs alliances. Il n'existe donc pas de chef chez les insectes et la fourmi doryle (bulumina) est à peine mentionnée, encore que sa dangerosité soit reconnue. Les forgerons (les clans Jorok, Venge) ont été écartés, minorés, oubliés. Et naturellement on ne reconnaît pas d'insectes forgerons. Toutefois le vieux refoulement de l'ancien pouvoir forgeron des siècles passés sur les rives du Logone se retrouve incidemment avec la luciole (wiwida) que l'on ne peut tuer car c'est un «forgeron-sorcier ». Les sorts jetés par les Masa passent encore par l'usage du fer et du charbon de bois (Tourneux et al. 1986 : 57).

Les Masa n'éprouvent pas le besoin de regrouper les insectes sociaux derrière un chef, hormis dans les jeux d'enfants où ils mettent en scène les personnages d'une chefferie de canton ou d'une mairie avec notables, secrétaires et factotum. On retrouve alors les mêmes criquets dans des rôles identiques : pour le chef Homoxyrrhepes punctipennis, pour son envoyé Cataloipes cymbiferus, le bas peuple étant représenté par de menus criquets. Quant aux bovins qui serviront au paiement des amendes, les petits criquets "bossus » comme Humbe tenuicornis feront l'affaire. On ne retrouve pas, non plus, les infinies relations avunculaires des montagnards. L'association mari/femme concerne, en revanche, nombre d'insectes. Ainsi le criquet bobota (Paracinema tricolor) est la femme du criquet bedemma, (Melis $2006: 69$ ). Quelques insectes retiennent l'attention, Pyrgomorpha vignaudii (tuvungudina) et Morphacris fasciata disposent de leur case dans la spirale divinatrice des devins, qui les utilisent couramment. En faisant tourner l'insecte autour de la tête et du corps du consultant malade, on fera endosser le mal par le criquet qui, une fois libéré, l'emportera en brousse. Une sauterelle verte à longues antennes (Ruspolia sp.), tlo ou tlogonda, devient chez les Masa un insecte personnalisé. On a même accolé à son nom un suffixe d'initié. Personnage clé des contes masa, c'est l'antihéros qui s'oppose constamment à hiya, l'écureuil fouisseur, le malin, alors que tlo est vantard, poltron et goinfre. Quant à sa première épouse, purkum, une autre sauterelle, il ne cesse de la maltraiter... Les Masa affirment qu'il n'existe pas de contes sans tlo (Melis 2006 : 387). Ce 
statut entraînerait la non-consommation de ces deux insectes par les adultes, ce qui est paradoxal car tlo est l'insecte le plus recherché pour ses qualités gustatives chez tous les groupes rencontrés ${ }^{8}$.

Figure 3

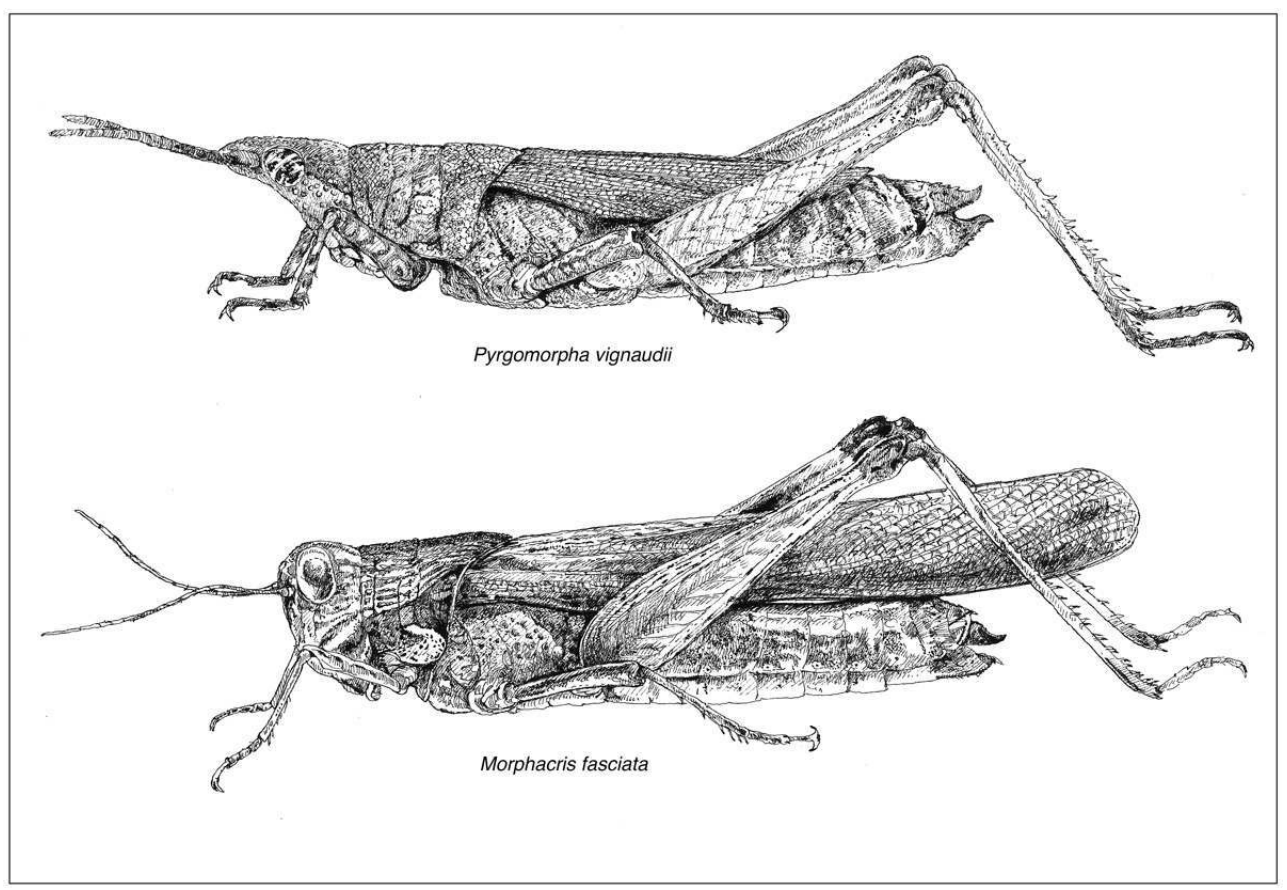

Dessin C. Seignobos

\section{Les Tupuri}

La concentration de pouvoirs autres que rituels est étrangère à la société tupuri précoloniale, mais elle ne manifeste aucune réticence vis-à-vis des forgerons. Comme chez leurs voisins masa, la classification des insectes n'existe pas, sauf dans les jeux d'enfants. La perception des Masa par les Tupuri est inversement proportionnelle à leur influence culturelle. Les Masa (jar baanre) ont apporté aux Tupuri la focalisation de leur société autour de la vache (dot, cure de lait (gurna), chants) et aussi leur second pilier social : l'initiation. Devant cette dette excessive, les Tupuri ont répondu par une inversion fantasmatique de leurs rapports. Tout ce qui vient des Masa est petit, imparfait, incompréhensible et ce dans tous les domaines. Cela se traduit naturellement dans la nomination des insectes, les petits, les difformes sont étiquetés "baanre» comme ceux vésicants ou urticants, par exemple la punaise verte Nezara viridula viridula (man cii baanre $=$ le sang des Masa).

Ces relations, pour le moins contrastées, entre les différentes communautés vis-à-vis de leurs insectes n'empêchent pas des comportements communs. Ils s'expriment dans le domaine, bien évidemment, de la consommation d'insectes, mais aussi dans celui des divertissements enfantins ou encore des soins et de la fabrication de charmes. 


\section{Quelques pratiques partagées concernant le monde des insectes}

Ces pratiques transcendent l'opposition montagne/plaine et celle animiste/musulmane. Tous les enfants jouent avec les mantes religieuses, qu'ils questionnent, les mantes étant réputées répondre par l'ouverture plus ou moins large de leurs pattes avant : «Bouba a-til un gros appétit?», «Est-il riche?", «A-t-il un gros sexe?». On confectionne des « avions » avec les cétoines en montagne (Lisle $1944: 65$ ) et, en plaine avec des libellules dont on a rogné les ailes ; attachées au bout d'un fil au poignet de l'enfant, elles amusent les tout petits pendant que leur mère continue à sarcler le champ. On arrache la tête de Mylabris spp. et, avec le jus irritant, on réalise des dessins sur le ventre et le visage. On prend la larve du fourmilion may maydo (fille + biceps en tupuri) pour la frotter à la saignée du bras, ce qui tétanise le muscle qui s'agite de mouvements incontrôlés, «c'est de la magie ", on peut évidemment poser des questions censées recevoir des réponses par ces diverses contractions. Les enfants jouent à l'enterrement avec des insectes qui représentent leurs nouveaux-nés morts et apprennent ce faisant les pleurs des deuils. Ce sont souvent de grosses fourmis comme Camponotus maculatus qui offre une coloration brun clair comme la peau des jeunes enfants. Ces jeux-apprentissages, rencontrés chez les Giziga, se déroulent également chez les Masa (Melis 2006 : 292).

Un certain nombre de pratiques ont pu se diffuser par voisinage. Des couples hommes/ femmes peuvent sceller une amitié ou un lien indéfectible en prenant un philtre confectionné à partir de punaises Dysdercus voelkeri (Pyrrhocoridae) qui cheminent souvent deux par deux. Ils peuvent aussi utiliser leur prédateur, plus gros, avec le même dessin blasonné, le réduve Phonoctonus lutescens car les populations ne les différencient pas. Ce même philtre peut également enduire le linteau de la porte de la case d'une femme afin de s'assurer de sa fidélité. Ces mêmes pratiques se retrouvent chez les Masa, les Gidar, les Peuls... 


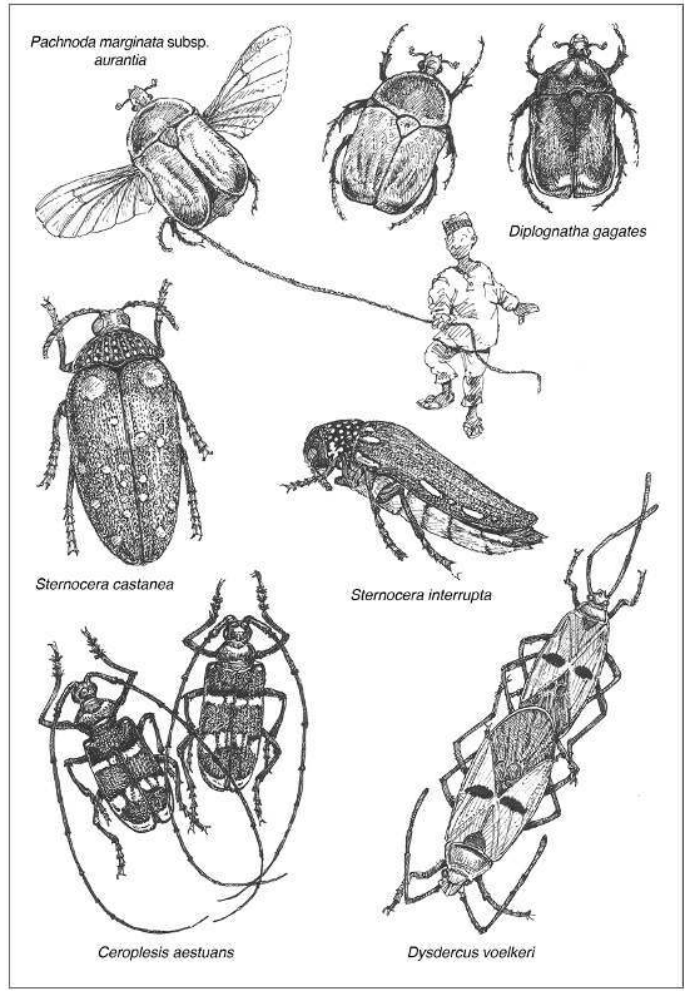

Dessin C. Seignobos

Si un insecte fait s'interroger toutes les populations de céréaliculteurs, c'est bien l'énorme charançon Brachycerus sacer, jeje (Mafa), mogwon (Mofu), skizan (Rhide), zoloda (traduit par « honte ») chez les Masa, kaye (Tupuri)... Appelé « l'insecte boudeur ", il entre en catalepsie quand on le touche. Il sectionne les attaches des grains de sorghos laissés à sécher dans les champs sans toutefois s'en emparer. Les paysans ne cessent de s'interroger : "pourquoi en veut-il à l'homme? ", «Le fait-il pour avoir des amis ou des clients parmi les fourmis? ». C'est l'exemple même de la "méchanceté gratuite ». Chez les Mofu, mogwon est surnommé magats (pince/ciseau) car sur l'aire de battage du mil il faut couper cet insecte en deux afin qu'il ne «fasse pas diminuer le mil» dans les silos, notion particulièrement prégnante pour les Mofu. Partout on utilise ce Brachyceridae comme « médicament » attaché au cou des enfants qui ont des difficultés de croissance, ne parlent pas, sont agités, tombent... Les Masa et Tupuri le considèrent comme un charme pour la protection de leur bétail. Il peut ainsi échapper aux voleurs en refusant de les suivre. On pourrait naturellement enchaîner les exemples sur les convergences dans les services susceptibles d'être rendus par les insectes aux hommes.

\section{Discussion}

Dans les monts Mandara les insectes livrent encore des présages et on les prend en compte, tout comme on se méfie des conséquences de certaines de leurs actions. Par exemple chez les Kapsiki, les Mofu et les Mafa, les branches tombées à terre à la suite d'un cravatage par les longicornes ne sauraient être utilisées pour les foyers sans exposer les enfants à des formes de nécroses; chez les Mafa cela rendrait les femmes stériles. On 
traite les insectes avec respect, même la fourmi réputée voleuse, la grosse Messor, n'est pas chassée des parcelles. On ne saurait se plaindre d'elle car lors de périodes de famine, on peut, en creusant son nid, en retirer une grosse calebasse de graines et puiser là les semences qui font défaut. Mettre le feu à leurs nids, comme cela se fait en plaine et pas seulement chez les musulmans, « offenserait Dieu car ce sont là des villages organisés à l'image de ceux des hommes ».

Certains insectes dont la "maison» et la progéniture ont été détruites non intentionnellement par l'homme peuvent se venger, comme pour madler papa, appelé faussement «punaise » chez les Mofu. Sa vengeance touche les femmes, qui avortent. En fait il s'agit là d'une réponse à l'angoisse de perdre ses enfants : c'est la faute de cet insecte comme, plus généralement, on dénonce l'engoulevent (Caprimulgus climacurus) dont on aurait, accidentellement, foulé la zone de ponte (Seignobos 2012). Il s'agit de la même démarche. On doit consulter un devin pour savoir qui doit officier pour enrayer la vindicte de l'insecte. On sacrifie une chèvre selon un protocole plus ou moins complexe, sur la « maison » détruite ou sur celle d'un autre madler papa et on y verse du rumen. Le devin reçoit une rétribution, renouvelée à la naissance du prochain enfant. Certains clans fournissent des devins spécialisés dans la lecture des signes donnés par les insectes, comme les Mokujek de Douvangar. Leur nombre ne cesse de diminuer aux dires mêmes de nos informateurs.

La désignation des insectes reflète les préoccupations des populations. La bonne protection des récoltes dans les greniers reste l'obsession des Mofu. Est alors indispensable une connaissance des plus fines des ravageurs et de leurs comportements et, à l'opposé, des insectes utiles (Seignobos et al. 1996: 140 et 148). Chez les Kapsiki méridionaux, où les densités de peuplement sont plus faibles et où les cultures sur le plateau semblent extensibles, l'entomofaune des silos perd de son importance.

40 Le monde des insectes pourrait encore se définir par rapport à une forme de « religion du mil » pour ces céréaliculteurs, entre ceux qui sont favorables à la croissance du mil et du sorgho et les accompagnent sans dégâts et ceux qui sont des ravageurs de mil potentiels ou réguliers. Le perce-oreille (Forficula senegalensis) ne gêne pas le mil tout en restant dans ses feuilles et ses panicules qui finissent leur maturité. Les Mafa le désignent comme keda ta daw (le « chien du mil »), les Peuls eux-mêmes reprennent cette appellation : rawandu gawri... À cela s'ajoutent certains hyménoptères. Les chenilles perforantes représentent, en revanche, les chefs de file des insectes destructeurs du mil.

41 Les Mofu, les Mafa, les Rhide et les Kapsiki forment une aire relativement homogène quant à l'intérêt porté aux insectes et aux projections sociales qu'ils en font. Le personnage central demeure la fourmi doryle et, à partir d'elle, s'organise la société des insectes. Plus on s'éloigne de ce foyer, plus cette qualification s'effiloche et la doryle perd de son importance. Après Gouria, chez les Kapsiki vers le sud, chez les Bana, dans ces contrées d'arbres mellifères (néré, Vitex spp.), la doryle est peu à peu supplantée par l'abeille et les ébauches de classification ressemblent plus à un jeu. On observe le même délaissement vers le nord, chez les Podokwo. Il existe donc des isolignes de croyance concernant les insectes. En plaine, hormis les Giziga proches des Mofu, nous avons mis en évidence l'absence de toute classification des insectes.

On peut d'ores et déjà émettre une hypothèse historique: le respect quasi religieux formulé par les Mofu vis-à-vis de leurs insectes en réfère directement à des pouvoirs rituels qui jadis réglaient leur vie économique et assuraient leur bien-être. Ce fut le cas de 
l'ancienne chefferie mowo Mbozom à laquelle succéda, sur les monts Mandara, celle apparentée de Gudur (xvIII ${ }^{\mathrm{e}} \mathrm{XIX} \mathrm{X}^{\mathrm{e}}$ siècles) (Seignobos 1991), l'une et l'autre situées dans la plus grande échancrure des monts Mandara. Postées ainsi à cette véritable «porte d'entrée de la montagne ", ces chefferies délivraient un quitus de passage à une infinité de groupes venant des plaines et qui gagneront ensuite différents reliefs. Les innombrables références à Gudur comme lieu d'origine signent l'un des fonds de peuplement les plus anciens des monts Mandara même si cette référence a pu parfois être usurpée. Ce centre de pouvoir pluriséculaire s'appuyait sur un arsenal de rituels censés assurer la protection des cultures, particulièrement les sorghos. Toutefois l'arrivée progressive de nouveaux venus de la plaine va marginaliser la chefferie de Mowo, déjà très exposée sur le piémont. Alors que celle de Gudur se maintenait et renforçait ses prérogatives dans la maîtrise des criquets et de la pluie, Mowo devait se recentrer sur les sacrifices de chenilles perforatrices, les noctuelles, comme défoliatrices, en particulier au cours de la petite saison sèche, au début des pluies (rhola en kapsiki, kwolay en mofu...). Les Mowo conservent encore aujourd'hui l'autorité pour officier lors d'invasions de chenilles sur les champs (Vincent 1991: 750 ; Seignobos 1995).

Les rituels concernant la maîtrise des insectes prédateurs révélaient encore au début du $\mathrm{xx}^{\mathrm{e}}$ siècle une grande complexité. Pour « gérer » les invasions acridiennes Gudur disposait à la fois d'une sorte de silo avec une porte en fer et un site, Husa, où se pratiquaient des sacrifices auprès d'anfractuosités d'où les essaims de criquets étaient censés partir ou qu'ils devaient intégrer. Lors d'attaques de criquets migrateurs, les populations touchées envoyaient une délégation à Gudur, avec des exemplaires de criquets vivants. Les devins les observaient et questionnaient les envoyés sur l'origine des vols, sur leurs trajectoires dans le ciel. Ces criquets, auxquels le ritualiste avait arraché de ses dents et les pattes et les mandibules, étaient jetés, mélangés à une bouillie de Crinum, dans le silo autel ou dans les crevasses de Husa. Pour les chenilles on constatait aussi chez les Mowo l'existence d'une case-autel où l'on jetait les chenilles prélevées sur les champs, avant d'officier sur les zones de cultures ravagées. Il existait également une anfractuosité dans le massif de Wazang-Morley, qu'il convenait de fermer afin de juguler ces invasions'.

Lors de mes enquêtes concernant la classification des insectes et le rôle de la fourmi doryle à Gudur même, mes informateurs cherchaient à éluder les questions, affirmant qu'ils chassent les insectes indésirables de leurs concessions non avec la doryle, mais avec la fourmi Messor... Manifestement la classification des insectes à partir de la fourmi doryle appartient aux Mowo et non à Gudur.

Bien peu de récits concernant les Mowo n'intègrent pas d'insectes. Nous ne donnerons qu'un exemple, une version de la genèse des Mowo dans leur départ du Wandala. Elle reprend la figure de mythe du vieux chef aveugle nourri par deux de ses femmes, mais la mère de l'aîné donne au chef des sauces de libellules (mbervek) alors que celle du cadet lui prépare une sauce aux sauterelles vertes (kwete kwete). La libellule a toujours été considérée comme l'insecte de l'indigent, du réprouvé, partout surnommée, comme en fflde, wala kuusel (sans viande). Alors que Ruspolia se présente comme l'insecte le plus recherché pour sa graisse et son goût surpassant celui de toute viande. Le chef bénit le fils de celle qui lui manifeste le plus d'attentions et il donnera au cadet les regalia de la chefferie... L'aîné, désavoué, doit fuir.

Les informateurs mowo ${ }^{10}$ fournissent à foison des rituels où interviennent des insectes. $\mathrm{Au}$ fur et à mesure que nos enquêtes progressaient il apparut que la première classification et caractérisation des insectes relevée chez les Mofu nord (Seignobos et al. 
1996) n'avait livré que des récits codés qui, en première lecture, ne laissaient voir qu'un jeu de comportements sociaux prêtés aux insectes. Cette classification fait référence en réalité à une charte de domination passée des Mowo dans la région qui fut autrement explicite.

Néanmoins un grand nombre de traits originels demeurent. Nomade, la fourmi doryle (de couleur rouge) représente les communautés mowo sans attache territoriale et aujourd'hui encore dispersées et inassimilables par les groupes au sein desquelles elles vivent. Sans armes, ni coercition guerrière les Mowo ont dominé la région au moins du $\mathrm{XVI}^{\mathrm{e}}$ au XIX ${ }^{e}$ siècle par le sacré et les rituels. Ils se revendiquent comme les représentants de Dieu sur terre, mieux encore les « fils de Dieu » (bizi ma bwi erlamhay). On comprend dès lors que les salutations adressées à Jaglavak (métaphore du pouvoir mowo) «mon prince » voire " mon Dieu », qui nous avaient semblé pour le moins excessives (Seignobos et al. 1996: 127, 128), avaient une autre destination. Une rencontre fortuite avec une colonie de fourmis doryles entraînait d'office un sacrifice. Lorsque l'on venait quémander, par le biais de prières, l'aide des doryles, on se devait de répandre sur la route «l'ocre qu'elles affectionnaient » et dont je ne comprenais guère l'utilité dans l'éradication des insectes de la maison, si ce n'est que jadis l'ocre était un monopole des Mowo pour leurs rituels. Le pouvoir prêté à ce petit insecte capable de détruire n'importe quelle créature, souvent durant son sommeil, en pénétrant par ses narines...semblait exorbitant. C'était encore là un pouvoir dont étaient crédités les chefs mowo. On peut également décrypter un certain nombre de comportements concernant d'autres insectes. Ndroa, par exemple, une Lepisiota, renvoie encore au pouvoir mowo. Cette fourmi arboricole vit surtout dans des Faidherbia albida, or cet arbre venu de la plaine sur les monts Mandara garda longtemps la réputation, au vu de l'étrangeté de sa phénologie, d'être la propriété des Mowo (et par la suite des chefs de massifs mofu, comme à Douvangar). Ainsi, piqué par ndroa le Mofu la salue d'un : «Envoyé de Dieu, merci ! ». D'autres insectes, comme singel gagazana, un Pheidole rouge représenterait une branche mowo car, comme jaglavak, il chasse (" donne des ordres ») aux autres insectes. Il est également susceptible de recevoir de l'ocre de la part des hommes...

Le rejet progressif et plus ou moins radical du pouvoir mowo/Mbozom dès la fin du XIX siècle va entraîner une péjoration de ses affidés, aujourd'hui des clans dispersés chez les Mofu, les Mafa et les Giziga. Tout un discours cherchant à les disqualifier socialement a encore cours et se répercute au niveau des alliances matrimoniales. Les Mowo, surtout leurs filles, sont difficilement épousables en raison de la réputation maléfique de leurs familles.

49 La désignation de certains insectes va participer de ce qui ressort d'une véritable « propagande » négative à leur endroit. Rappelons que la couleur des Mowo est le rouge. La chenille défoliatrice du sorgho, de couleur brique (Diacrisia sp.) est le plus souvent désignée ou surnommée " mowo ». L'insecte le plus dangereux pour le mil du grenier est un petit coléoptère rouge de la famille des Tenebrionidae (Palorus subdepressus), que les Mofu appellent sla wandala (la vache du Wandala), à partir du nom de louange même des Mowo revendiquant leur origine du Wandala. Chez les Mafa (à Shigoulé, Magoumaz et Vouzad), ce ténébrion est désigné par le nom même de mbozom. Un Polydesmidae rouge avec des anneaux plus clairs (Habrodesmus duboscqui), capable d'opérer des rassemblements importants suivis de gros dégâts sur les cultures est également désigné comme mbozom, toujours chez les Mafa. Signalons que les Mafa se sont, dans le passé, débarrassés avec violence de la tutelle religieuse mowo-Mbozom. 
50 Par ailleurs on trouve à Massakal, Wazan, Douroum et dans la région, des traces d'une sorte de « contrepropagande » ou plus vraisemblablement des bribes de l'ancien discours officiel où la fourmi doryle est identifiée aux Mowo. On lui prête la possession de la Pierre de la guerre (pour faire cesser les conflits) et elle se voit désignée comme protecteur des récoltes, depuis le battage jusqu'aux silos où elle concourt à «faire augmenter le mil ». Certains Mofu continuent à enfermer une fourmi doryle sur leur aire de battage lors du vannage du mil. Ce sont là les lointains avatars d'une longue lutte idéologique mettant en scène les insectes.

51 Le modèle de classification à partir de son prince, la fourmi doryle, qui a pris forme à l'époque de la domination mowo n'a pas, par la suite, été écarté, mais a survécu comme référent social inaltérable indépendamment des changements de pouvoir.

Outil social, politique, didactique, voire ludique, le monde des insectes servira-t-il encore longtemps des sociétés qui migrent vers les villes, se scolarisent et manifestent une vie religieuse (islam/christianisme) de plus en plus démonstrative?

\section{BIBLIOGRAPHIE}

Aberlenc H.-P. \& Deguine J.-P. 1999 - Les insectes des monts Mandara : le regard des MofuDiamaré et le regard de l'entomologiste. In Baroin C. \& Boutrais J. (Ed.) L'homme et l'animal dans le bassin du lac Tchad. Paris, IRD : 109-131. (Colloques et Séminaires).

Barreteau D. 1999 - Les Mofu-Gudur et leurs criquets. In Baroin C. \& Boutrais J. (Ed.) L'homme et l'animal dans le bassin du lac Tchad. Paris, IRD : 133-169. (Colloques et Séminaires).

Lisle M. de 1944 - Note sur la faune coléoptérologique du Cameroun. Bulletin de la Société d'Etudes Camerounaises 5, mars : 55-71.

Melis A. 2006 - Dictionnaire masa-français. Dialectes Gumay et Haara (Tchad). Sassari, Democratia Sarda, $293 \mathrm{p}$.

Seignobos C. 1991 - Le rayonnement de la chefferie théocratique de Gudur (Nord-Cameroun). In Boutrais J. (Ed.) Du politique à l'économique, études historiques dans le bassin du lac Tchad. Vol. III. Paris, Orstom : 225-315.

Seignobos C. 1995 - Introduction à l'histoire de Mowo. In Iyebi Mandjek O. \& Seignobos C. Terroir de Mowo, saturation foncière et émigration. Dpgt/Sodecoton/Orstom : 69-79.

Seignobos C. 1999 - Jaglavak, prince des insectes chez les Mofu du Nord-Cameroun. In Baroin C. \& Boutrais J. (Ed.) L'homme et l'animal dans le bassin du lac Tchad. Paris, IRD : 299-304. (Colloques et Séminaires).

Seignobos C. 2012 - L'engoulevent ou l'étrangeté porteuse de malheur (Nord du Cameroun. Revue d'éthnoécologie en ligne 1. http ://ethnoecologie.revues.org/690.

Seignobos C., Deguine J.-Ph. \& Aberlenc H.-P. 1996 - Les Mofu et leurs insectes. Journal d'Argic. Trad. et de Bota. Appl. XXXVIII (2) : 125-187.

Tourneux H., Seignobos C. \& Lafarge F. 1986 - Les Mbara et leur langue (Tchad). Paris, Selaf, 317 p. 
Vincent J.-F. 1991 - Princes montagnards du Nord-Cameroun. T1 et T2. Paris, l'Harmattan, 774 p.

\section{NOTES}

1. Nous remercions Henri-Pierre Aberlenc, entomologiste (Cirad), pour sa relecture attentive.

2. Rappelons que la notion d'« insectes » est généralement absente. Les informateurs mofu, par exemple, dans le souci de trouver un équivalent au français, avancent pour désigner les insectes terrestres, tout ce qui grouille comme les larves de moustiques, matel gwadeng, ou ce qui semble innombrable; maneneh (maa nane hay: qui/nombreuses/familles), appellation des termites Microcerotermes ou ont-ils recours à des onomatopées.

3. En Mofu Gudur : mapela pela, en kapsiki : palpa ou pulpa, qui désignera ensuite la bible, le livre, dont les pages tournées renvoient au papillon pliant ses ailes. Chez les Masa, c'est kibirkibira et chez les Tupuri, gibir-gibir. Même les Peuls ont adopté le redoublement : mallum mallum hon (pl.), yel (petit)...

4. Dans les monts Mandara, chez les Mofu nord, les déterminations ont été réalisées entre 1992 et 1996 par les entomologistes H.-P. Aberlenc et J.P. Deguine (Cirad). Toujours dans les Mandara, au cours d'une mission de juin 2011, chez les Kapsiki, les entomologistes Philippe Le Gall (IRD) et Bernard Lassalle ont assuré les déterminations. De notre côté nous présentions à nos informateurs rhide, wula et mafa les collections d'insectes du laboratoire de l'IRA (Institut de Recherche Agronomique à Maroua), accompagné du technicien entomologiste Alioum Mana. Nous avons repris cette démarche en plaine chez les Masa, Muzuk et Tupuri.

5. Les Giziga Bwi Marva reconnaissent à la même fourmi doryle jaglavak, qu'ils dénomment mugurmak, un rôle bénéfique et protecteur des récoltes et ils l'utilisent également pour chasser les termites des habitations. Ils n'en font pas, en revanche, la clef de voûte d'une classification du monde des insectes.

6. Bien que les mantes religieuses (Dictyoptera mantodea), Mugidaba chassent et dévorent les criquets, elles restent hors classement, comme non historisées, réduites à des «hyènes du criquet » (motkoya hoyok).

7. Nous n'avons fait qu'effleurer le monde polysémique des criquets et qu'entrevoir sa charge cognitive pour les Giziga Bwi Marva. Une détermination plus couvrante et plus précise des Acrididae giziga aiderait encore à sa compréhension.

8. Cette Rupsolia (mekotkote) peut parfois constituer un interdit majeur, comme pour les ressortissants du clan du chef giziga bwi marva, sous peine de faire mourir le chef.

9. Dans la région comprise entre Maroua et les monts Mandara, toutes les chenilles (Spodoptera exempta, Diacrisia sp., Amsacta sp....) sont dites go'da maavahay (les choses de Mowo).

10. Kadakay Kadanga, Udlabudlay Sumaka (Bwi Morley), Girgdam Matarpa, Bi Taklo Akle, Kutkobi Ritju (Mowo)...

\section{RÉSUMÉS}

Cet article est le prolongement de celui publié par le JATBA 38 de 1996 : «Les Mofu et leurs insectes ", de C. Seignobos, J.-Ph. Deguine et P.H. Aberlenc. Il répond à une interrogation : la focalisation de la société mofu sur son entomofaune se retrouve-t-elle chez ses voisines des 
monts Mandara ? Et qu'en est-il du regard sur les insectes des populations des plaines?

Le biais des insectes dans l'étude des sociétés est toujours révélateur de leur histoire et de leur organisation sociale et politique. Toutefois la classification des insectes en parallèle avec la société des hommes n'est attestée que sur les monts Mandara. Elle est particulièrement prégnante dans la zone d'influence des populations mowo, initiatrices de la chefferie de Gudur ( $\mathrm{XVI}^{\mathrm{e}}$-début du $\mathrm{Xx}^{\mathrm{e}}$ siècle). Les Mowo prétendaient « régner " par des rituels assurant le contrôle de la pluie comme celui des insectes ravageurs des cultures.

This article is conceived as an extension of that published in JATBA 38, 1996, "Les Mofu et leurs insectes ", by C. Seignobos, J.-Ph. Deguine and P.H. Aberlenc. It aims at answering the following question: is the Mofu's particular interest in their insects also found among their neighbours in the Mandara mountains? And what about the views on insects among the people of the plains? Tackling society through insects is always indicative of their history and their social structure. However, the classification of insects according to human society is only attested in the Mandara mountains, particularly in the sphere of influence of the Mowo people, the alleged founders of the Gudur chiefdom (16th-beginning of the 20th cent). The Mowo even claimed to " reign » by the means of rituals allowing them to control the rain as well as crop pests.

\section{INDEX}

Mots-clés : insectes/sociétés, nord Cameroun, monts Mandara, plaine du Logone, chefferie Mowo-Gudur

Keywords : insects/society, Northern Cameroon, Mandara mountains, Logone's flood plain, Mowo-Gudur chiefdom

Index géographique : Cameroun

Population Mowo

\section{AUTEUR}

\section{CHRISTIAN SEIGNOBOS}

Géographe, directeur de recherche IRD émérite 